\title{
Context and alcohol consumption behaviours affect inhibitory control
}

Adam W Qureshi $(\mathrm{PhD})^{1 *}$, Rebecca L Monk $(\mathrm{PhD})^{1}$, Charlotte R Pennington $(\mathrm{PhD})^{2}$, Xiaoyun Li $(\mathrm{PhD})^{1} \&$ T. Leatherbarrow $(\mathrm{BSc})^{1}$

${ }^{1}$ Edge Hill University, St Helens Road, Ormskirk, UK, L39 4QP

${ }^{2}$ University of the West of England, Coldharbour Lane, Bristol, UK, BS16 1QY

*Corresponding author: Adam W. Qureshi, E-mail: qureshia@edgehill.ac.uk

Tel +44 (0)1695 584 498, Fax: 44(0)1695 579997

Funding: This work was supported by Alcohol Research UK (ARUK; grant number SG 14/15 203, 2014 - 2015). The funders had no role in study design, data collection and analysis, decision to publish, or preparation of the manuscript.

Word count: 3907 


\begin{abstract}
Contrasting the traditional focus on alcohol-related visual images, this study examined the impact of both alcohol-related auditory cues and visual stimuli on inhibitory control (IC). Fifty-eight participants completed a Go/No-Go Task, with alcohol-related and neutral visual stimuli presented with or without short or continuous auditory bar cues. Participants performed worse when presented with alcohol-related images and auditory cues. Problematic alcohol consumption and higher EC were associated with better IC performance for alcohol images. It is postulated that those with higher EC may be better able to ignore alcohol-related stimuli, whilst those with problematic alcohol consumption are unconsciously less attuned to these. This runs contrary to current dogma and highlights the importance of examining both auditory and visual stimuli when investigating IC.
\end{abstract}

Keywords: Inhibitory control; context effects; alcohol-related visual and auditory stimuli 
Context and alcohol consumption behaviours affect inhibitory control

\section{Introduction}

Alcohol consumption is one of the top five global risk factors for disease, disability and mortality (World Health Organisation, 2014) and its approximate cost to the UK is $£ 21$ billion per annum (Health and Social Care Information Centre, 2015). It has also been linked with wider socio-cultural problems, including alcohol related crime and reduced productivity, costing the UK over $£ 18$ billion per annum (Institute of Alcohol Studies, 2013). Whilst alcohol consumption has many antecedents, it is a commonly held belief that alcohol consumption may be driven, at least in part, by limitations in inhibitory control (IC) - the ability to control or suppress pre-potent responses (de Wit, 2009). Accordingly, problem alcohol consumption has been associated with impaired IC (c.f. Field et al., 2010; Goudriaan, Oosterlaan, De Beurs \& van den Brink, 2006; Lawrence, Luty, Bogdan, Sahakian \& Clark, 2009), suggesting that problems with alcohol consumption may be driven by a reduced ability to control impulses. Similarly, increases in alcohol consumption in non-clinical samples have also been linked with reductions in IC in response to alcohol-related stimuli (c.f. Christiansen, Cole, Goudie \& Field, 2012; Murphy \& Garavan, 2011; Nederkoorn, Baltus, Guerrieri \& Wiers, 2009). As such, increasing our study of IC may be critically important to developing our understanding of the drivers of alcohol consumption in both clinical and nonclinical samples. This understanding may also inform the development of intervention approaches (c.f. Jones et al., 2016), the success of which would have clear and powerful social applications.

IC has been suggested to be a limited, consumable resource, which is depleted in situations that involve resisting temptation (Muraven \& Baumeister, 2000; though see Leotti \& Wegner, 2009 for a trait approach). Akin to mental fatigue, fluctuations in IC can be influenced by one's environment or context and changes in motivation/ priorities 
(Inzlicht \& Berkman, 2015). Particular contexts may therefore require differing levels of IC, contingent on the salience of environmental cues.

Questionnaire research has shown that people's self-reported alcohol consumption and associated behaviour (i.e., impulse control) varies as a function of environmental context (Nyaronga, Greenfield, \& McDaniel, 2009; Monk \& Heim, 2013a; 2013b; 2014).

Overcoming the limitations of self-reports, research employing indirect measures also indicates that alcohol-related cognitions alter in the presence of real or laboratorymanipulated contextual cues (Cox et al., 1999; Read \& Curtin, 2007; Monk et al., 2016; Wall, Mckee, \& Hinson, 2000). For example, alcohol-related stimuli have been found to heighten physiological arousal, alcohol craving and actual consumption across both clinical and nonclinical samples (Albery et al., 2015; Field et al., 2014; Kaplan et al., 1985; Nees et al., 2012; Papachristou et al., 2012; Roberts, Miller, Weafer, \& Fillmore, 2014). This research converges to suggest that contextual factors may have a manifest impact on IC, with the presence of such cues in the immediate environment presenting as a risk factor for alcohol consumption behaviour.

IC has been measured widely using the Go/No-Go Association Task (GNG; Nosek \& Banaji, 2001), which requires participants to respond to frequent 'go' stimuli ( $80 \%$ of trials) and inhibit their responses to infrequent 'no-go' stimuli ( $20 \%$ of trials). For example, participants may be presented with letters on the screen, and asked to respond to every letter except "K". As the 'go' stimuli are more frequent, responding becomes the dominant action. The participant's ability to (not) respond correctly when presented with the infrequent "K" ('no-go') stimulus is therefore a measure of their response control or IC (though see Suskauer et al., 2008). Studies have shown that heavy drinkers respond faster when alcohol-related 'go' stimuli are used, and make more commission errors (false alarms, e.g. they respond) when no-go stimuli are super-imposed onto alcohol-related images (Kreusch, Vilenne, \& 
Quertemont, 2013; Petit, Kornreich, Noël, Verbanck, \& Campanella, 2012). However, this prior research predominantly compares appetitive alcohol-related cues with non-appetitive neutral cues (e.g. a stapler - Kreusch et al., 2013). Resultantly, it could be argued that any observed response differences may be due to the contrast between the appetitive and nonappetitive nature of the cues, rather than their association with alcohol per se (see Adams et al., 2013).

Research employing the Cued Go-No-Go task has examined whether the introduction of neutral visual cues prior to the presentation of a target impacts inhibitory control. Here Marczinski, Combs and Fillmore (2007) found that individuals who were classified as binge drinkers exhibited greater inhibitory impairment in response to moderate doses of alcohol compared to non-binge drinkers. Research using alcohol-related visual stimuli (e.g. Kreusch et al., 2013; Weafer \& Fillmore, 2012) also suggests that alcohol-related cues may impact impulsivity by triggering schemas driving arousal and behaviour (Tiffany, 1990). Whilst hitherto applied solely to visual cues, classic conditioning ${ }^{1}$ and relational frame principles ${ }^{2}$ would suggest that the same processes might also apply to auditory cues (Riecke, SchultePelkum, Caniard, \& Bülthoff, 2005). Further, the cumulative use of auditory and visual stimuli may create a more immersive and ecologically valid effect, cueing participants to the types of real-world factors which may affect responses, albeit whilst situated in a controlled laboratory (Monk \& Heim, 2013b).

Alcohol-related auditory cues (e.g. opening of a beer can; as compared to neutral stimuli such as opening a door) have been shown to increase general craving in alcoholics (Heinze, Wölfling, \& Grüsser, 2007). Other research has also examined the impact of

\footnotetext{
${ }^{1}$ The theory that the continual pairing of a stimulus with a specific response/outcome is seen to cause this stimulus to become conditioned/associated with an outcome or response.

${ }^{2}$ The theory that via the repeated pairing of stimuli with responses, people learn that certain things are associated and, thus, these things become related in memory.
} 
relevant and irrelevant novel auditory cues on general GNG performance (Leiva, Parmentier, Elchlepp, \& Verbruggen, 2015). Results indicate that relevant novel cues enhanced no-go performance (with cues as the no-go signal), whereas irrelevant novel cues impaired performance (when cues were presented before stimuli). This supports a stimulus-detection account of reorienting attention and response inhibition, in that auditory cues may impair or bolster inhibitory control, depending on their relation to the visual stimuli presented in the task. This research gives weight to the assertion that attention is oriented to novel or unexpected events, which impairs no-go performance when these events are irrelevant but enhances no-go performance when they are relevant (Leiva, et al., 2015). However, to our knowledge, no research has examined the concurrent use of both alcohol-related visual and auditory stimuli on inhibitory control processes.

\subsection{Research Overview \& Hypotheses}

The current study sought to examine whether alcohol-related visual and auditory stimuli may make people respond more or less impulsively, and whether this pattern is different for those with lower effortful control and higher levels of problematic alcohol consumption. In particular, this study sought to examine university students in light of cross cultural observations which place this population at particular health-related risks resulting from their engagement in frequent and heavy episodic drinking, or binge drinking (e.g., Gordon, Heim, \& MacAskill, 2012; Wechsler \& Kuo, 2000). To this end, the first aim of the current study was to examine the effects of alcohol-related visual and auditory cues on inhibitory control utilising a Cued Go/No-Go Association Task. It was predicted that participants would be more error prone when responding to alcohol-related stimuli, shown by increased false alarms for 'no-go' alcohol stimuli. According to the stimulus-detection model ${ }^{3}$, performance

\footnotetext{
${ }^{3}$ In the GNG task, response selection occurs in conjunction with response inhibition (Suskauer et al., 2008). In the current study, the auditory stimuli can be compatible or incompatible with the following visual stimuli in
} 
would be expected to improve if participants associated alcohol-related visual stimuli with auditory cues, due to semantic linkage. However, if auditory cues are treated as irrelevant, as they have no relation to the task requirements, then they should impair performance. Moreover, previous research has utilised short bursts of sound to examine the effect of auditory stimuli on inhibitory control (Leiva et al., 2015), and it could be argued that continuous alcohol-related cues may be more representative of a real life situation (e.g., being in a bar), and hence more ecologically valid. Whilst hitherto unexplored, it was therefore postulated that long alcohol-related cues would result in manifestly different patterns of responding in contrast to short cues, due to possible effects of habituation on impulsivity (c.f., Givois \& Pollack, 2000).

The second aim was to examine whether self-reported alcohol consumption and trait effortful control moderated the effect of alcohol-related visual stimuli and auditory cues on inhibitory control. Previous research has indicated that participants who reported higher and problematic alcohol consumption show a heightened approach bias towards alcohol-related stimuli (Albery et al., 2015; Field et al., 2014; Roberts et al., 2014). Problem consumption has also been related to dysfunctions in brain regions associated with attention and response inhibition, leading to a reduced ability to suppress responses (Ahmadi et al., 2013). In line with this, it was predicted that individuals with higher AUDIT scores would display a higher false alarm rate (FAR) on alcohol-related no-go trials. Given that effortful control is likely to be negatively related to AUDIT scores (c.f., Ahmadi et al., 2013), it was also predicted that participants with lower effortful control and problematic alcohol consumption would show

respect of alcohol context, and so the speed of response selection may be faster when they are compatible. Once the response is selected, it then needs to be executed, which will, in the case of no-go trials, require response inhibition. This may result in overall faster response times and accuracy to go stimuli, and likewise, due to faster/more efficient stimuli recognition, lower false alarm rates. Additionally, the current study utilises multiple go and no-go stimuli, hence increasing the response selection requirement. There are also other factors linked to impulsivity in substance misuse (De Wit, 2009), but this study focuses on inhibitory control. 
higher false alarm rate (FAR) on no-go trials, whereas those with higher effortful control would display better performance. See Table 1 for example FAR predictions.

\section{INSERT TABLE 1 HERE}

\section{Method}

\subsection{Participants}

Decisions regarding sample size were based on a power analysis of pilot research and recruitment was stopped once a sample of 60 participants had been met through online signups to the study. Participants were assigned equally and randomly to one of two experimental conditions, in which they heard either short or long auditory cues (in order to assess whether brief auditory cues are sufficient to impact responses or whether sustained auditory cues, which are more immersive, are required). Fifty-eight university students (Mage $=21.95, S D=$ 6.29, range 18-63 years, 34 female) entered the final analyses, of which none reported visual acuity or auditory deficits. Two participants' data were excluded because their AUDIT scores represented outliers, with scores over 30. Mean AUDIT scores were slightly above the cut-off for clinical assessment (scores of 8 or above; Babor et al., 2001). Nevertheless, they are comparable with recent research using UK student samples (e.g., Clarke, Field, \& Rosa, 2015). Boxplots indicated that there were other no outliers on the remaining measures (dependent variables on the Cued Go/No-Go Task and Effortful Control).

\subsection{Measures}

The Alcohol Use Disorders Identification Test (AUDIT; Saunders et al., 1993) was used to assess hazardous drinking patterns (Cronbach's $\alpha=0.83$ ). 
The Effortful Control sub-scale ${ }^{4}$ of the Adult Temperament Questionnaire (ATQ; Rothbart, Ahadi, \& Evans, 2000) was used to measure participants' ability to regulate their behavior (Cronbach's $\alpha=.87$ ). Higher scores represent higher trait effortful control, and research has shown that effortful control measures are highly correlated with response inhibition (Pandey et al., 2012).

\subsection{Stimuli}

The Cued Go/No-Go Task was programmed with E-Prime 2.0. Alcohol-related visual stimuli consisted of 5 images of bottles of unbranded beer, and these were contrasted with 25 orange juice bottles. Neutral visual stimuli consisted of 5 water bottle images, and these were contrasted with 25 empty bottles (See Figure 1). Target stimuli were matched for palatability, size, luminosity and orientation.

Auditory cues were delivered via headphones whilst participants completed the task. Participants were exposed to either a long auditory bar cue $(44.14 \mathrm{kHz})$ or a short auditory cue $(48 \mathrm{kHz})$ during testing. These were clipped using the software Audacity from audio recorded in a bar/restaurant in the USA and uploaded free of copyright to YouTube (https://youtu.be/jAg6tyC9Xxc). On trials or blocks with no bar cue, no sound was heard. The task was divided into 16 blocks, eight with standard instructions and eight with reversed instructions. Incorrect responses were followed by a feedback tone of $250 \mathrm{~ms}$. The standard and reversed blocks were split equally into neutral and alcohol-related visual stimuli blocks, totalling 1,120 trials. Target stimuli were presented pseudo-randomly in $20 \%$ of trials for each block, and block order was also randomised.

\footnotetext{
${ }^{4}$ This sub-scale incorporates questions on inhibitory control (capacity to suppress inappropriate approach behaviour), activation control (capacity to perform an action when there is a strong tendency to avoid it) and attentional control (capacity to focus attention as well to shift attention when desired)
} 


\section{INSERT FIGURE 1 HERE}

The internal reliabilities of the stimuli were calculated, and are shown in Table 2 for the standard design. Due to the low number of target stimuli, reliability values of 0.4 were considered acceptable (c.f. Williams \& Kaufman, 2012). Reliabilities for the reversed design are shown in Table 3.

\section{INSERT TABLES 2 and 3 HERE}

Pilot research $(n=50)$ was conducted over numerous iterations to establish the optimum alcohol-related auditory cues. Specifically, participants were asked to rate a series of alcohol-related sounds. The short and long cues with the highest overall ratings were used in the present study ${ }^{5}$. A second pilot study also employed an alcohol-related cue (pub), a neutral cue (office/work environment), and no cue. Findings indicated that there were no differences in inhibitory performance between the 'neutral' social auditory cue and no auditory cue. There was, however, an apparent impairment in inhibitory performance to the 'alcohol-related' social auditory cue (pub) compared to the same neutral social auditory cue. These pilot results suggested that any observed difference in inhibitory control-related performance were not the product of drawing comparisons between any noise versus no noise and, rather, reflected an effect of alcohol-related auditory cues ${ }^{6}$. Therefore, for the current study, the neutral cue was removed to simplify the design and increase statistical power.

\footnotetext{
${ }^{5} 82 \%$ of participants rated the bar cue as being of a social environment involving alcohol consumption. This was in line with procedures by Heinze et al. (2007).

${ }^{6}$ Additional information on the methodology and results of pilot studies can be found in Supporting Files 1 (Pilot study details), 2 (GNAT (Initial pilot data).xlsx) and 3 (GNAT (Second pilot data).xlsx).
} 
Visual stimuli were also piloted to ensure the palatability, size and luminosity and orientation were matched.

\subsection{Procedure}

Following briefing and the provision of consent, participants sat in a quiet room in front of a computer screen and wore headphones. After instructions, participants were given 20 practice trials (removed from the final analyses). During standard trials, participants were instructed to respond to 'go' targets (orange juice bottles, empty bottles) and inhibit their respond to 'no-go' targets (beer bottles, water bottles). For reversed trials, participants were instructed to respond only to the no-go stimuli.

In the short auditory cue condition, a bar cue was presented randomly upon the onset of the fixation cross for $500 \mathrm{~ms}$ and the cues were balanced so that they preceded $50 \%$ of the go- and no-go trials. The presentation order for short cue trials is shown in Figure 2. For the long cue condition, the bar cue was played from the beginning of $50 \%$ of the blocks until their end. Participants then completed the AUDIT and ATQ as the final component of the study in order to limit the signal strength of the study, where participants infer the aims of the study and behave accordingly (Davies \& Best, 1996).

\section{INSERT FIGURE 2 HERE}

\subsection{Analytic Strategy}

Statistical analyses were carried out using SPSS 22.0. Initial mixed-design ANOVAs were conducted for standard and reversed designs, with within-subject factors of visual stimuli (alcohol-related and neutral images) and auditory cue type (bar and none) and a betweensubjects factor of auditory cue length (long- and short-cue group). The main dependent variable of interest was False Alarm Rates (FARs) on no-go trials (\%). Further results (Go 
Response Times and Go Accuracy) can be found in Appendix 3. Main effects and interactions were elucidated using Bonferroni-corrected pairwise comparisons, and a total of 1,040 trials from each participant entered the final analyses.

Three-way mixed ANCOVAs were then conducted to elucidate the moderating role of alcohol consumption and effortful control. Total AUDIT scores were included as a covariate to control for participants' problematic alcohol consumption in light of research showing that heavy drinking is associated with deficits in response inhibition (Nederkoorn et al., 2009). Effortful control was also included as a covariate to control for any individual or group differences in trait inhibition. Simple main effects were used to analyse any moderating effects of AUDIT and effortful control, an adaption of the method suggested by Judd, Kenny and McClelland (2001; see Appendix 1).

\section{Results}

\subsection{Participant characteristics}

A series of independent $t$-tests indicated that there were no significant differences between participants' demographics, and self-reported AUDIT and ATQ scores as a function of auditory cue length (short vs. long-cue). All descriptive statistics are shown in Appendix 2.

\subsection{FAR}

In the standard ${ }^{7}$ design there was a main effect of visual stimuli, with FARs higher for alcohol-related stimuli, $F(1,58)=16.62, p<0.01, \eta_{p}^{2}=0.22$. There was also a main effect of auditory cue type, with FARS higher when the bar cue was present, $F(1,58)=20.19, p<$

\footnotetext{
${ }^{7}$ The reversed design had no significant main effects or interactions with and without AUDIT and Effortful Control as covariates.
} 
$0.01, \eta_{p}^{2}=0.26$. There was a significant interaction between visual stimuli and auditory cue length, $F(1,58)=4.76, p=0.03, \eta_{p}^{2}=0.22$. For the short cue group, FARs were higher for alcohol-related compared to neutral stimuli $(p<0.01)$, but there was no difference for the long cue group. Simple main effects showed that there was no difference in FARs between the short-cue and long-cue groups for either the alcohol-related or neutral visual stimuli. For the reversed design there were no significant main effects or interactions.

When AUDIT and Effortful Control were added as covariates to the standard block analyses, there were no significant main effects. As there was no main effect of visual stimuli, as in the ANOVA, these results suggest that problematic alcohol consumption and trait effortful control may explain the impaired performance in response to alcohol-related visual stimuli. There was, however, a significant interaction between auditory cue type and auditory cue length, $F(1,50)=4.63, p=0.04, \eta_{p}^{2}=0.09$ (Figure 3 ). Whilst there were no differences in FAR between short and long cue conditions ( $p$ 's $>0.51)$, both the short and long cue groups had higher FARs when the bar cue was presented compared to no cue (both $p$ 's $<0.05)$, equivalent to the main effect in the initial ANOVA.

\section{INSERT FIGURE 3 HERE}

There was a significant interaction between auditory cue type, auditory cue length and Effortful Control, $F(1,50)=5.63, p=0.02, \eta_{p}^{2}=0.10$. To assess this interaction, Effortful Control was held at low and high values of 40 and 60 respectively (as the mean value was 49.68). At low levels of Effortful Control, higher FARs were found when the bar cue was presented for the long cue group (compared to no cue; $p<0.01, \eta_{p}^{2}=0.17$ ). At high levels of Effortful Control, no differences were found though the pattern suggests that better trait effortful control was associated with better inhibitory performance (Figure 4). 


\section{INSERT FIGURE 4 HERE}

There was also a significant interaction between visual stimuli, auditory cue length, AUDIT and Effortful Control, $F(1,50)=4.30, p=0.04, \eta_{p}^{2}=0.08$. Here AUDIT scores were held at 8 (scores of 8 and below are deemed as low risk drinking) and 16 (scores of 16 and above are categorised as harmful $(<20)$ and dependent $(>19))$, with Effortful Control again at 40 and 60. Participants with low AUDIT scores had higher FARS for alcohol visual stimuli, regardless of their effortful control levels (both $p$ 's $<0.05$ ). At high AUDIT and low Effortful Control, the same pattern was shown $(p<0.05)$. Participants with high AUDIT scores and high effortful control did not show this responding pattern (all $p$ 's $>.05$; Figure 5). This suggests that those with problematic alcohol consumption but high effortful control are able to respond more accurately to alcohol-related visual stimuli. There were no differences between the auditory cue length groups, $p>.05$.

\section{INSERT FIGURE 5 HERE}

\subsection{Summary of results}

Higher effortful control was associated with greater accuracy for both neutral and alcoholrelated visual stimuli, but accuracy was always lower for alcohol-related, relative to neutral, visual stimuli. Alcohol-related auditory cues also decreased accuracy. The main effects of visual stimuli and auditory cue type were not present when AUDIT and Effortful Control were added as covariates, suggesting that problematic alcohol consumption and inhibitory control are involved in these apparent effects on IC. However, including AUDIT and Effortful Control as covariates resulted in interactions that may suggest that problem alcohol 
consumption and trait inhibitory control may be more important determinants on the effect of visual stimuli on inhibitory control, whilst the effect of auditory cues may be more independent of such factors. Importantly, the general pattern of lower accuracy for alcoholrelated visual stimuli did not hold when both AUDIT and Effortful Control scores were high, with no difference between visual stimuli type. This may suggest that when effortful control is high, those with problematic alcohol consumption are better able to control their responses and ignore potentially distracting alcohol-related stimuli.

\section{Discussion}

The current research builds on the existing literature by introducing auditory alcohol-related cues alongside visual stimuli in a traditional Go/No-Go Association Task. Whilst visual stimuli may be a proxy for assessing the effect of different contexts on inhibition, the inclusion of auditory cues may provide a more immersive method for testing variability in inhibitory control. Consistent with hypotheses, results of the current research suggest that alcohol-related pictorial stimuli and auditory alcohol-related cues can make people respond more impulsively, resulting in more errors of inhibition. This is in line with prior research (Kreusch et al., 2013; Petit et al., 2012; Weafer \& Fillmore, 2012) which suggests that the presence of alcohol-related stimuli may distract attention and diminish inhibitory control.

Moreover, participants found it harder to inhibit responses to alcohol-related stimuli when cued with short auditory bar cues. This finding is consistent with theories of priming and cue reactivity (Roberts et al. 2014; Heinze et al., 2007; c.f also Verbruggen \& Logan, 2007), suggesting that alcohol-related pictures and auditory sounds may serve as a distraction, heightening task demands and diminishing inhibitory control. However, this effect was not demonstrated for those who were exposed to continuous blocks of bar-related 
sound. It is plausible that short bursts of alcohol-related sound may be more distracting and lead to more commission errors relative to continuous auditory cues, which are more immersive in nature. Previous research has demonstrated the impact of alcohol-related auditory sounds on alcohol craving (Heinze et al., 2007). However, this is the first study to examine the impact of visual and auditory alcohol-related stimuli on inhibitory control, highlighting the importance of further research into this area.

Problem alcohol consumption and trait effortful control also appeared to moderate the effect of alcohol-related stimuli on inhibitory control. At both low and high AUDIT levels, participants responded less accurately towards alcohol relative to neutral stimuli. The inclusion of trait effortful control changed these findings. Participants with high AUDIT scores and low levels of effortful control had higher FARS towards alcohol-related stimuli. However, more hazardous consumption (as indicated by the AUDIT) and higher control was associated with lower errors to alcohol-related visual stimuli. This may suggest that effortful control enables those with more problematic consumption to regulate their behaviour, perhaps through a more efficient response selection process (Weafer \& Fillmore, 2012).

In the long-cue condition, participants with lower levels of effortful control showed higher FARS towards alcohol-related stimuli presented with a bar-related auditory cue. Yet, there was no difference found for those with higher effortful control, suggesting they may be better able to ignore potential contextual distractions. Effortful control may thus reduce the negative effect of auditory alcohol-related cues on performance, by allowing participants to regulate their responses to external stimuli (Rothbart et al., 2000). This seems particularly plausible given that effortful control incorporates measures of both heightened attention and inhibition traits, which would seem to convey a performance advantage when faced with irrelevant cues that may otherwise detract attention (Leiva et al., 2015). 
Differences between the current study and that of previous research may be explained by the stimuli used, and the introduction of auditory cues. For example, the current study included both alcohol-related and neutral appetitive pictorial stimuli, whereas past research has used non-appetitive neutral objects (e.g., a stapler; Kreusch et al., 2013) as the non-target stimuli. It is plausible that diminished inhibitory control towards alcohol-related stimuli in such studies may be due to the appetitive nature of such cues, rather than their association to alcohol. Second, there were more non-target stimuli utilised compared to previous studies on inhibitory control (c.f., Pennington, Qureshi, Monk, \& Heim, 2016). Tasks requiring response selection in addition to response inhibition may require additional processing and thus result in slower responses and greater chance of errors (Simmonds et al. 2008). Third, whilst prior research has paid attention to the moderating role of alcohol consumption and effortful control, these variables tend to be studied independently of another (c.f., Heinze et al., 2007). The current study considered the interaction of both trait effortful control and alcohol consumption, and argues that individual variations in these factors may moderate inhibitory control and explain divergent findings in the literature. Finally, in previous studies, the provision of alcohol-related questions prior to testing may have primed participants (as noted by Kreusch and colleagues (2013), whilst in this study this was avoided.

\subsection{Limitations and Conclusions}

University students are immersed in a social, pub-based drinking culture (c.f. Straus \& Bacon, 1953) where there are strong normative influences on drinking (e.g., Ahern Galea, Hubbard, Midanik, \& Syme, 2008). Indeed, although there may be differences in the nature and/or manifestations of student drinking culture across different institutions and across varying regions, it is apparent that the values, expectations and norms inferred by this culture can have a powerful potential to impact the amount and nature of university -based drinking (c.f., Martin \& Zamboanga, 2017). It should therefore be noted that context related cueing 
might be more likely in at least some of the current university sample (c.f. Rumelhart \& Todd, 1993). Further research beyond this sample is thus recommended. It has also been noted with concern that self-report measures such as the AUDIT (Babor et al., 2001) are susceptible to social desirability (c.f. Davies \& Best, 1996; Davis et al., 2010; Williams \& Nowatzki, 2005). The present research offers evidence of a complex relationship between alcohol-related stimuli, response selection and response inhibition. Here it is suggested that stimuli variations may highlight the complexity of research in this area. Thus, whilst we advocate for further research to test these assertions, the current results should be considered as a first step in advancing in the study of alcohol-related inhibition.

In an innovative approach, this study has examined the effect of both alcohol-related visual stimuli and auditory cues on response inhibition and selection. The present findings suggest that alcohol-related stimuli may make impulse control more difficult. This apparent variation in inhibitory control may be caused by changes in attentional bias and response selection but appears to be moderated by both problematic alcohol consumption and trait effortful control. As such, cue dependent reductions in inhibitory control appear to be less likely in those who report more problematic alcohol use but have higher trait levels of effortful control, somewhat contrary to current dogma, highlighting the importance of examining both auditory and visual stimuli. The present findings offer important caveats to existing research findings in the field. They may also inform interventions which seek to reduce alcohol consumption by training people to improve their IC and thus potentially reduce their consumption (c.f. Jones \& Field, 2013). In order to be effective, inhibitory control training methods must be aware of, and be able to respond to, context-dependent fluctuations in disinhibition, whilst also considering individual-level alcohol consumption and trait inhibitory control. The current research may consequently have key implications for intervention approaches which aim to reduce problem levels of alcohol consumption. 
Specifically, they suggest that training methods may also wish to consider the inclusion of other alcohol-related modalities such as sounds (or even smells c.f. Monk, Sunley, Qureshi, \& Heim, 2016), in light of their potential to impact IC. 
Context \& Alcohol Consumption Behaviour Affect IC

\section{Table headings}

\section{Table 1}

Example FAR and Go Trial predictions by AUDIT and Effortful Control.

\section{Table 2.}

GNG visual stimuli reliability by auditory cue (standard design).

Table 3 .

GNG visual stimuli reliability by auditory cue (reversed design). 


\section{Figure captions}

\section{Figure 1.}

Experimental stimuli (Top: L-R; beer (target), juice (non-target), beer (target), juice (nontarget); Control stimuli (Bottom: L-R; water (target), empty bottle (non-target), empty bottle (non-target), water (target).

Figure 2.

Go/No-Go trial procedure (short-cue, standard, target stimulus).

Figure 3.

FAR by auditory cue and auditory cue length (error bars are $95 \%$ confidence intervals).

Figure 4.

FAR for bar cue and no cue (standard design) by auditory cue length (Effortful Control as covariate).

Figure 5.

FAR for No-Go trials by AUDIT, EC and auditory cue length, for visual stimuli; error bars are confidence intervals). 


\section{References}

Adams, S., Ataya, A. F., Attwood, A. S., \& Munafò, M. R. (2013). Effects of alcohol on disinhibition towards alcohol-related cues. Drug \& Alcohol Dependence, 127, 1-3, 137142.

Ahern, J., Galea, S., Hubbard, A., Midanik, L., \& Syme, S. L. (2008). "Culture of drinking” and individual problems with alcohol use. American journal of epidemiology, 167(9), 1041-1049.

Ahmadi, A., Pearlson, G. D., Meda, S. A., Dager, A, Potenza, M. N, Rosen, R., . . Stevens, M. C. (2013). Influence of alcohol use on neural response to go/no-go task in college drinkers. Neuropsychopharmacology, 38, 2197-2208.

Albery I. P., Sharma, D., Noyce, S., Frings, D., \& Moss A. C. (2015). Testing a frequency of exposure hypothesis in attentional bias for alcohol-related stimuli amongst social drinkers. Addictive Behaviors Reports. 1, 68-72.

Babor, T. F., Higgins-Biddle, J. C., Saunders, J. B., \& Monteiro, M. G. (2001). The Alcohol Use Disorders Identification Test, Guidelines for Use in Primary Care (2nd ed.). World Health Organization: Department of Mental Health and Substance Dependence.

Christiansen, P., Cole, J. C., Goudie, A. J. \& Field, M. (2012). Components of behavioural impulsivity and automatic cue approach predict unique variance in hazardous drinking. Psychopharmacology, 219(2): 501 - 10

Clarke, N. C., Field, M., \& Rose, A. K. (2015). Evaluation of a brief personalised intervention for alcohol consumption in college students. PLoS One, 10, e0131229. 
Cox W. M., Yeates, G.N., \& Regan, C. M. (1999). Effects of alcohol cues on cognitive processing in heavy and light drinkers. Drug \& Alcohol Dependence, 55(1-2), 85-89.

Davies, J. B., \& Best, D. W. (1996). Demand characteristics and research into drug use. Psychology \& Health, 11, 291-299.

Davis, C. G., Thake, J., \& Vilhena, N. (2010). Social desirability biases in self-reported alcohol consumption and harms. Addictive Behaviors, 35, 302-311.

de Wit, H. (2009). Impulsivity as a determinant and consequence of drug use: a review of underlying processes. Addiction Biology, 14(1): 22 - 31

Field, M., Wiers, R. W., Christiansen, P., Fillmore, M. T. \& Verster, J. C. (2010). Acute Alcohol Effects on Inhibitory Control and Implicit Cognition: Implications for Loss of Control Over Drinking. Alcoholism Clinical \& Experimental Research, 34(8): 1346 - 1352

Field, M., Marhe, R., \& Franken, I. H. (2014). The clinical relevance of attentional bias in substance use disorders. CNS spectrums, 19(03), 225-230.

Givois, V., \& Pollack, G. S. (2000). Sensory habituation of auditory receptor neurons: implications for sound localization. Journal of Experimental Biology, 203(17), 2529-2537.

Gordon, R., Heim, D., \& MacAskill, S. (2012). Rethinking drinking cultures: A review of drinking cultures and a reconstructed dimensional approach. Public health, 126(1), 311.

Goudriaan, A. E., Oosterlaan, J., de Beurs, E. \& van den Brink, W. (2006). Neurocognitive functions in pathological gambling: a comparison with alcohol dependence, Tourette syndrome and normal controls. Addiction, 101(4), 534 - 47 
Health and Social Care Information Centre, (2015). Statistics on Alcohol; England, 2015 (pp. 1 - 54). Health and Social Care Information Centre. Retrieved from http://content.digital.nhs.uk/catalogue/PUB17712/alc-eng-2015-rep.pdf

Heinze, M., Wölfling, K., \& Grüsser, S. M. (2007). Cue-induced auditory evoked potentials in alcoholism. Clinical Neurophysiology, 118, 856-862.

Inzlicht M., \& Berkman, E. (2015). Six questions for the resource model of self-control (and some answers). Social \& Personality Psychology Compass, 9, 511-524.

Jones, A., Di Lemma, L. C. G., Robinson, E., Christiansen, P., Nolan, S., Tudur-Smith, C. \& Field, M. (2016). Inhibitory control training for appetitive behaviour change: A metaanalytic investigation of mechanisms of action and moderators of effectiveness. Appetite, 97: $16-28$

Jones, A., \& Field, M. (2013). The effects of cue-specific inhibition training on alcohol consumption in heavy social drinkers. Experimental and Clinical Psychopharmacology, 21(1), 8-16.

Judd, C. M., Kenny, D. A., \& McClelland, G. H. (2001). Estimating and testing mediation and moderation in within-subjects designs. Psychological Methods, 6(2), 115-134.

Kaplan, R. F., Cooney, N. L., Baker, L. H., Gillespie, R. A., Meyer, R. E., \& Pomerleau, O. F. (1985). Reactivity to alcohol-related cues: Physiological and subjective responses in alcoholics and nonproblem drinkers. Journal of Studies for Alcohol and Drugs, 46, 267272.

Kreusch, F., Vilenne, A., \& Quertemont, E. (2013). Response inhibition toward alcoholrelated cues using an alcohol go/no-go task in problem and non-problem drinkers. Addictive Behaviors, 38(10), 2520-2528. 
Lawrence, A. J., Luty, J., Bogdan, N. A., Sahakian, B. J. \& Clark, L. (2009). Impulsivity and response inhibition in alcohol dependence and problem gambling. Psychopharmacology, 207(1): $163-72$

Leiva, A., Parmentier F. B., Elchlepp, H. \& Verbruggen, F. (2015). Reorienting the mind: The impact of novel sounds on go/no-go performance. Journal of Experimental Psychology: Human Perception \& Performance. 41(5), 1197-1202.

Leotti, L. A., \& Wafer, T. D. (2009). Motivational influences on response inhibition. Journal of Experimental Psychology: Human Perception \& Performance, 36, 430-447.

Marczinski C. A., Combs, S. W., \& Fillmore, M. T. (2007). Increased sensitivity to the disinhibiting effects of alcohol in binge drinkers. Psychology of Addictive Behaviors, $21(3), 346-354$.

Martin, J. L., \& Zamboanga, B. L. (2017). Putting multisite college alcohol research into context: A call to assess the drinking culture on college campuses. Addictive Behaviors. Early online

Monk, R. L., \& Heim, D. (2014). A real-time examination of context effects on alcohol cognitions. Alcoholism: Clinical and Experimental Research, 38, 2452-2459.

Monk, R. L., \& Heim, D. (2013a). Environmental context effects on alcohol-related outcome expectancies, efficacy and norms: A field study. Psychology of Addictive Behaviors, 27, 814-818.

Monk, R. L., \& Heim, D. (2013b). Panoramic projection; affording a wider view on contextual influences on alcohol-related cognitions. Experimental \& Clinical Psychopharmacology, 21, 1-7. 
Monk, R.L., Sunley, J., Qureshi, A.W., \& Heim, D. (2016). Smells like inhibition: The effects of olfactory and visual alcohol cues on inhibitory control. Psychopharmacology, 233, 1331-1337

Muraven, M., \& Baumeister R. F. (2000). Self-regulation and depletion of limited resources: Does self-control resemble a muscle? Psychological Bulletin, 126, 247-259.

Murphy, P. \& Garavan, H. (2011). Cognitive predictors of problem drinking and AUDIT scores among college students. Drug and Alcohol Dependence, 115(1-2): 94 - 100

Nederkoorn, C., Baltus, M., Guerrieri, R., \& Wiers, R. W. (2009). Heavy drinking is associated with deficient response inhibition in women but not in men. Pharmacology Biochemistry \& Behavior, 93(3), 331-336.

Nees F., Diener, C., Smolka, M. N., \& Flor, H. (2012). The role of context in the processing of alcohol-relevant cues. Addiction Biology, 17, 441-451.

Nosek B. A., \& Banaji, M. R. (2001). The go/no-go association task. Social Cognition, 19(6), 625-666.

Nyaronga, D., Greenfield, T. K., \& McDaniel, P. A. (2009). Drinking context and drinking problems among black, white, and Hispanic men and women in the 1984, 1995, and 2005 U.S. National Alcohol Surveys. Journal of Studies on Alcohol \& Drugs, 70, 16-26.

Pandey, A. K., Kamarajan, C., Tang, Y., Chorlian, D. B, Roopesh, B. N, Manz N., . . Porjesz, B. (2012). Neurocognitive deficits in male alcoholics: An ERP/sLORETA analysis of the N2 component in an equal probability Go/NoGo Task. Biological Psychology, 89(1), 170-182. 
Papachristou, H., Nederkoorn, C., Havermans, R., van der Horst, M., \& Jansen, A. (2012). Can't stop the craving: the effect of impulsivity on cue-elicited craving for alcohol in heavy and light social drinkers. Psychopharmacology, 219(2), 511-518.

Pennington, C. R., Qureshi, A., Monk, R. L., \& Heim, D. (2016). The effects of stereotype threat and contextual cues on alcohol users' inhibitory control. Addictive Behaviors, 54, $12-17$.

Petit G., Kornreich, C., Noël, X., Verbanck, P., \& Campanella, S. (2012). Alcohol-related context modulates performance of social drinkers in a visual Go/No-Go task: A preliminary assessment of event-related potentials. PLoS ONE, 7(5), 1-11.

Read, J. P., \& Curtin, J. J. (2007). Contextual influences on alcohol expectancy processes. Journal of Studies on Alcohol and Drugs, 68, 759-770.

Riecke, B. E., Schulte-Pelkum, J., Caniard, F., \& Bülthoff, H. H. (2005). Influence of auditory cues on the visually-induced self-motion illusion (circular vection) in virtual reality. Proceedings of $8^{\text {th }}$ international workshop on Presence, 49-57.

Roberts, W., Miller, M. A., Weafer, J., \& Fillmore, J. (2014). Heavy drinking and the role of Inhibitory control of attention. Experimental \& Clinical Psychopharmacology, 22(2), 133140.

Rothbart, M. K, Ahadi, S. A., \& Evans, D. E. (2000). Temperament and personality: Origins and outcomes. Journal of Personality \& Social Psychology, 78(1), 122-135.

Rumelhart D. E., \& Todd, P. M. (1993). Learning and connectionist representations. In D. E Meyer \& S. Kornblum (Ed.), Attention and performance XIV: Synergies in experimental psychology, artificial intelligence, and cognitive neuroscience (pp. 3-30). Cambridge: MIT Press. 
Saunders, J.B., Aasland, O.G., Babor, T.F., De la Fuente, J.R., \& Grant, M. (1993). Development of the alcohol use disorders identification test (AUDIT). WHO collaboration project. Addiction, 88, 791-804.

Simmonds D. J., Pekar, J. J., Mostofsky, S. H. (2008) Meta-analysis of Go/No-go tasks demonstrating that fMRI activation associated with response inhibition is taskdependent. Neuropsychologia, 46, 224-232.

Straus R., \& Bacon, S. (1953). Drinking in college. Yale University Press: New York.

Suskauer, S. J., Simmonds, D. J., Fotedar, S., Blankner, J. G., Pekar, J. J., Denckla, M. B. \& Mostofsky, S. H. (2008). Functional magnetic resonance imaging for abnormalities in response selection in attention deficit hyperactivity disorder: differences in activation associated with response inhibition but not habitual motor response. Journal of Cognitive Neuroscience, 20, 478 - 493.

Tiffany S. T. (1990). A cognitive model of drug urges and drug-use behavior: role of automatic and non automatic processes. Psychological Review, 97, 147-168.

Verbruggen, F., \& Logan, G. D. (2007). Automatic and controlled response inhibition: Associative learning in the Go/No-go and Stop-signal paradigms. Journal of Experimental Psychology: General, 137, 649-672.

Wall, A. M., Mckee, S. A., \& Hinson, R. E. (2000). Assessing variation in alcohol outcome expectancies across environmental context: An examination of the situational-specificity hypothesis. Psychology of Addictive Behaviors, 14, 367-375.

Weafer J., \& Fillmore, M. T. (2012). Alcohol-related stimuli reduce inhibitory control of behavior in drinkers. Psychopharmacology, 222, 489-498. 
Wechsler, H. \& Kuo, M. (2000). College students define binge drinking and estimate its prevalence: Results of a national survey. Journal of American College Health, 49(2), $57-$ 64.

World Health Organisation (2014). Global status report on alcohol and health 2015. Geneva: WHO Press.

Williams B. J., \& Kaufman, L. M. (2012). Reliability of the Go/No-Go Association Task. Journal of Experimental Social Psychology, 48(4), 879-891.

Williams, R. J., \& Nowatzki, N. (2005). Validity of adolescent self-report of substance use. Substance Use \& Misuse, 40, 299-311. 